\title{
Molecular Detection, Phylogenetic Analysis, and Identification of Transcription Motifs in Feline Leukemia Virus from Naturally Infected Cats in Malaysia
}

\author{
Faruku Bande, ${ }^{1,2}$ Siti Suri Arshad, ${ }^{1}$ Latiffah Hassan, ${ }^{3}$ and Zunita Zakaria ${ }^{1}$ \\ ${ }^{1}$ Department of Veterinary Pathology and Microbiology, Faculty of Veterinary Medicine, Universiti Putra Malaysia, \\ 43400 UPM Serdang, Selangor, Malaysia \\ ${ }^{2}$ Department of Veterinary Services, Ministry of Animal Health and Fisheries Development, PMB 2109, Usman Faruk Secretariat, \\ 840221 Sokoto, Sokoto State, Nigeria \\ ${ }^{3}$ Department of Veterinary Laboratory Diagnostics, Faculty of Veterinary Medicine, Universiti Putra Malaysia, \\ 43400 UPM Serdang, Selangor, Malaysia
}

Correspondence should be addressed to Siti Suri Arshad; suri@upm.edu.my

Received 26 June 2014; Revised 11 September 2014; Accepted 11 September 2014; Published 17 November 2014

Academic Editor: Philip H. Kass

Copyright (c) 2014 Faruku Bande et al. This is an open access article distributed under the Creative Commons Attribution License, which permits unrestricted use, distribution, and reproduction in any medium, provided the original work is properly cited.

\begin{abstract}
A nested PCR assay was used to determine the viral RNA and proviral DNA status of naturally infected cats. Selected samples that were FeLV-positive by PCR were subjected to sequencing, phylogenetic analysis, and motifs search. Of the 39 samples that were positive for FeLV p27 antigen, 87.2\% (34/39) were confirmed positive with nested PCR. FeLV proviral DNA was detected in 38 (97.3\%) of p27-antigen negative samples. Malaysian FeLV isolates are found to be highly similar with a homology of $91 \%$ to $100 \%$. Phylogenetic analysis revealed that Malaysian FeLV isolates divided into two clusters, with a majority (86.2\%) sharing similarity with FeLV-K01803 and fewer isolates (13.8\%) with FeLV-GM1 strain. Different enhancer motifs including NF-GMa, Krox-20/WT1Idel2, BAF1, AP-2, TBP, TFIIF-beta, TRF, and TFIID are found to occur either in single, duplicate, triplicate, or sets of 5 in different positions within the U3-LTR-gag region. The present result confirms the occurrence of FeLV viral RNA and provirus DNA in naturally infected cats. Malaysian FeLV isolates are highly similar, and a majority of them are closely related to a UK isolate. This study provides the first molecular based information on FeLV in Malaysia. Additionally, different enhancer motifs likely associated with FeLV related pathogenesis have been identified.
\end{abstract}

\section{Introduction}

Feline leukaemia virus (FeLV) is a gammaretrovirus associated with anaemia, immunodeficiency, leukaemia, and lymphoma in cats [1]. FeLV has been studied extensively as a model for human immunodeficiency virus (HIV) and human T-cell lymphoma virus (HTLV) infections [2]. FeLV is distributed worldwide; however, prevalence varies greatly with geography and with risk factors that include age, health status, and population density [3,4]. A recent study reported FeLV seroprevalence of $5.1 \%$ and $18.9 \%$ in healthy and sick Malaysian cats, respectively [4]. On the other hand, studies carried out in other Asian regions reported 0\% FeLV prevalence in Vietnam [5]; 14.7\% among cats in Singapore [6]; 2.9\% in Japan [7]; and 6\% each from Taiwan and Thailand $[8,9]$. In non-Asian countries, FeLV prevalence has been reported to be $4.8 \%$ on Prince Edward Island, Canada [10]; 5.3\% and $3.7 \%$ in Raleigh and Gainesville, USA, respectively [11]; 3.4\% in all Canada [12]; and 4.6\% in Egypt [13]. These discrepancies in prevalence of FeLV may arise due to differences in cat's lifestyle and FeLV vaccination practices in different countries [4].

Diagnosis of FeLV is usually performed by detection of p27 antigen [14]. However, demonstrating p27 antigen is difficult during early viraemia and with latent infections. Studies have shown that FeLV viral RNA and provirus DNA are better predictors of progressive and latent infections, respectively $[15,16]$. 
Apart from the envelope gene of FeLV, the long terminal repeats (LTRs) play important role in determining disease outcome and in differentiating exogenous from endogenous FeLV $[15,17]$. Vaccination against FeLV is not carried out in Malaysia and, to date, FeLV clinical status of Malaysian cats has not been investigated using molecular assays. Additionally, unlike the ubiquitous feline infectious peritonitis (FIP) [18] sequence and phylogenetic characteristics of the Malaysian FeLV isolates have not been elucidated. The objectives of this study are to evaluate the presence of FeLV viral RNA and provirus DNA in selected antigenaemic and nonantigenaemic cats, respectively. Sequence characteristics, enhancer motifs as well as phylogenetic relationships of the Malaysian FeLV also were determined.

\section{Materials and Methods}

2.1. Animals and Sampling. Heparinized blood samples were collected from cats presented at University Veterinary Teaching Hospital, Universiti Putra Malaysia (UVH-UPM). The samples were tested for the presence of FeLV p27 viral antigen using a commercially available test kit [4]. These cats were divided into p27 antigen positive and p27 antigen-negative groups. From each group, 39 cats were selected by convenience sampling method and the samples were subjected to PCR analysis. All cats had no history of vaccination against FeLV as vaccination against FeLV is not practiced in Malaysia. All samples were collected by the attending veterinary clinicians, as part of routine practices. In addition, consent for evaluation was obtained from the cat owners, prior to sampling.

2.2. Nucleic Acid and PCR Amplification. Viral RNA was extracted from the plasma of p27-positive cats, using high pure viral RNA purification kit (Roche, Germany). On the other hand, genomic DNA was isolated from whole blood of p27-negative cats, using QIAGEN DNA extraction kits (QIAGEN, Germany). All nucleic acid extraction procedures were carried out according to manufacturers' instructions. RNA was reverse transcribed and subjected to nested PCR, using a one-step access RT PCR (Promega, USA). Genomic DNA was amplified by nested PCR assay.

Two sets of primers (outer and inner primers) were synthesised (1st BASE, Malaysia) and used to amplify a $601 \mathrm{bp}$ segment of FeLV-U3LTR and partial gag regions. This segment recognises exogenous but not endogenous FeLV segments presence in cat genome; thus the primers used in this study are specific for exogenous FeLV detection. Outer PCR reaction was carried out using U3-F(1) (5'-ACA GCA GAA GTT TCA AGG CC $\left.-3^{\prime}\right)$ and G-R(1) (5'-GAC CAG TGA TCA AGG GTG AG-3') primers. The inner PCR reaction was carried out with $\mathrm{U} 3-\mathrm{F}(2)\left(5^{\prime}\right.$-GCT CCC CAG TTG ACC AGA GT- $3^{\prime}$ ) and G-R(2) (5'-GCT TCG GTA CCA AAC CGA AA-3') primers [15].

The PCR mixture was prepared in $25 \mu \mathrm{L}$ reaction volume containing $10 \mathrm{mM}$ each of dNTPs mix, $0.2 \mathrm{mM}$ Tfl DNA polymerase $(5 \mathrm{U} / \mu \mathrm{L}), 0.1 \mathrm{U}$ AMV $(5 \mathrm{U} / \mu \mathrm{L}), 0.1 \mathrm{U}$ recombinant RNasin ribonuclease inhibitor $(400 \mathrm{U} / \mu \mathrm{L}), 0.8 \mathrm{U} \mathrm{MgSO}_{4}$
(25 mM), $20 \mathrm{pmol}$ of each of the forward and reverse primer, $5.0 \mu \mathrm{L}$ of 1 times buffer, and $1 \mu \mathrm{L}$ RNA or DNA template. Nuclease-free water was used to bring the mixture to its final volume of $25 \mu \mathrm{L}$. AMV reverse transcriptase enzymes and RNasin ribonuclease inhibitor were included only when RNA was a starting template for the PCR assay. In the nested PCR step, $1 \mu \mathrm{L}$ of outer PCR product was used as template.

In both inner and outer PCR steps, the target gene regions were amplified using the following conditions: reverse transcription: $45^{\circ} \mathrm{C}$ ( $45 \mathrm{~min}$ ) (only in the case of RNA), initial denaturation: $94^{\circ} \mathrm{C}(2 \mathrm{~min})$, denaturation: $94^{\circ} \mathrm{C}(45 \mathrm{sec})$, annealing: $58^{\circ} \mathrm{C}(30 \mathrm{sec})$, extension: $72^{\circ} \mathrm{C}(1 \mathrm{~min}), 35$ cycles of repeats, and final extension: $72^{\circ} \mathrm{C}(7 \mathrm{~min})$. PCR product was electrophoresed using $1.5 \%$ agarose (SeaKem LE USA), stained with $0.5 \mu \mathrm{g} / \mathrm{mL}$ ethidium bromide (Bio-Rad USA), and visualised under UV light (Geldoc system, Bio-Rad, USA). Extraction and amplification procedures were carried out in separate hood to reduce chances of contamination.

2.3. Sequence and Phylogenetic Analyses. In order to gain insight on the characteristics of Malaysian FeLV sequences, 29 nested PCR-positive samples (RNA $n=14$; DNA provirus $n=15$ ) were selected and purified using an Accuprep purification kit (Bioneer, Daejeon, Korea). Sequencing was carried out based on the amplified U3LTR-gag segment using a standard ABI Big Dye terminator version 3.1 sequence kit (Applied Biosystem). The obtained sequences were analysed for homology using the NCBI Basic Local Alignment Search Tool (BLAST: http://www.ncbi.nlm.nih.gov). In addition, multiple sequence alignment was carried out using ClustalW and the percentage nucleotide identity was determined using DNA identity matrix $[19,20]$. On the other hand, single nucleotide polymorphism (SNP), DNA distance matrix, and transcription binding proteins prediction analyses were carried out using geneious software version R7 [20]. A neighbour-joining (NJ) phylogenetic tree was constructed based on the U3LTR-gag sequences using MEGA5 software. The tree reliability was assessed using 100 bootstrap replicates [21]. All nucleotide sequences were deposited with the NCBI GenBank (Table 1).

\section{Results and Discussion}

FeLV infection is of concern to cat owners due to its ability to induce tumours and immunodeficiency, thus predisposing cats to other secondary diseases. In this study, a U3-LTR and gag regions of exogenous without endogenous FeLV sequences were amplified by nested PCR methods. Post-PCR analysis using electrophoresis revealed an expected amplicon size of $770 \mathrm{bp}$ in the outer PCR and $601 \mathrm{bp}$ in the nested inner PCR assay (Figure 1). Overall, it was found that $97.4 \%$ (38/39) of p27 antigen-negative cats were positive for FeLV provirus DNA suggesting that this category of cats likely goes undetected when only p27 detection is used to judge their FeLV clinical status. Similar studies reported high prevalence of FeLV provirus DNA in Brazilian cats [22]. However, Hofmann-Lehmann et al. [23] reported a lower provirus DNA rate in cats in Switzerland. The observed differences 


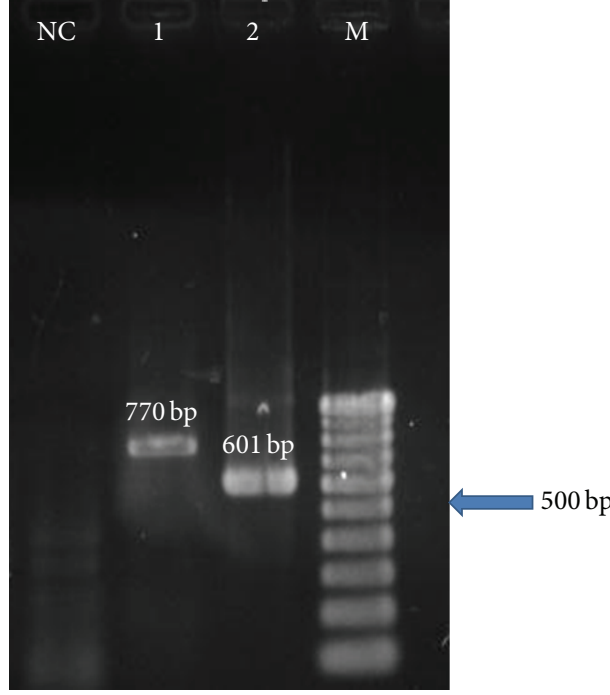

FIGURE 1: Nested RT-PCR assay used to detect FeLV U3LTR-gag sequence from naturally infected cats in Malaysia. The outer PCR reaction was performed using primer pair U3-F(1) and G-R(1) and amplified $770 \mathrm{bp}$ (lane 1) whilst inner PCR reaction was performed using primer pair U3-F(2) and G-R(2) and amplified $601 \mathrm{bp}$ (lane 2). Lane NC: negative control, lane M: 100 bp DNA marker (iDNA, Malaysia).

in prevalence among different countries could be associated with cat lifestyle, as well as variations in factors known to favour FeLV transmission [3, 4]. Provirus DNA detection rate observed in this study could be associated with regressive or latent FeLV infection, which is characterized by integration of DNA provirus into the host cell genome and absence of viral antigen in circulation $[1,15]$.

The consequence of latent FeLV infection is that provirus DNA could reactivate to an infectious state, especially following stress and/or immunosuppression. Thus, cats that are p27 antigen-negative, but provirus DNA positive, could serve as sources of infection of FeLV-naïve cats [24]. A previous study has established an association between feline lymphoma and provirus DNA positivity in p27 antigen-negative cats, though this has not been evaluated in the present study [25]. Moreover, transmission of FeLV has been shown to occur in cats following blood transfusion from cats with provirus DNA, thus highlighting the importance of screening blood donor cats for provirus DNA [26].

Viral RNA was detected in $87.2 \%$ (34/39) of p27 antigenpositive cats whereas $13 \%$ (5/39) tested negative using RTPCR assay. Since plasma viral RNA is an indicator of FeLV viraemia, cats that are positive for FeLV p27 antigen and viral RNA are likely to harbour replicating virus [27]. Cats in this category may progress to a persistent viraemic stage, succumbing to FeLV-associated illness [28].

Failure to detect FeLV viral RNA in about 13\% p27 antigen-positive cats (p27-positive/viral RNA-negative) could result from atypical infection, wherein the virus is sequestered and replicates locally in tissues such as salivary gland, mammary gland, and urinary epithelium, causing intermittent or low-grade antigenaemia, although there is no
TABLE 1: List of local and reference sequences used in phylogenetic analysis of Malaysian FeLV isolates.

\begin{tabular}{|c|c|c|c|}
\hline Isolate & Accession number & Country & Source \\
\hline FeLV-UPM01 & HQ197367 & Malaysia & This study \\
\hline FeLV-UPM02 & HQ197368 & Malaysia & This study \\
\hline FeLV-UPM03 & HQ197369 & Malaysia & This study \\
\hline FeLV-UPM04 & HQ197370 & Malaysia & This study \\
\hline FeLV-UPM05 & HQ197371 & Malaysia & This study \\
\hline FeLV-UPM06 & HQ197372 & Malaysia & This study \\
\hline FeLV-UPM07 & HQ197373 & Malaysia & This study \\
\hline FeLV-UPM08 & HQ197374 & Malaysia & This study \\
\hline FeLV-UPM09 & HQ197375 & Malaysia & This study \\
\hline FeLV-UPM10 & HQ197376 & Malaysia & This study \\
\hline FeLV-UPM11 & HQ197377 & Malaysia & This study \\
\hline FeLV-UPM12 & HQ727890 & Malaysia & This study \\
\hline FeLV-UPM13 & HQ727891 & Malaysia & This study \\
\hline FeLV-UPM14 & HQ727892 & Malaysia & This study \\
\hline FeLV-UPM15 & JF815538 & Malaysia & This study \\
\hline FeLV-UPM16 & JF815539 & Malaysia & This study \\
\hline FeLV-UPM17 & JF815540 & Malaysia & This study \\
\hline FeLV-UPM18 & JF815541 & Malaysia & This study \\
\hline FeLV-UPM19 & JF815542 & Malaysia & This study \\
\hline FeLV-UPM20 & JF815543 & Malaysia & This study \\
\hline FeLV-UPM21 & JF815544 & Malaysia & This study \\
\hline FeLV-UPM22 & JF815545 & Malaysia & This study \\
\hline FeLV-UPM23 & JF815546 & Malaysia & This study \\
\hline FeLV-UPM24 & JF815547 & Malaysia & This study \\
\hline FeLV-UPM25 & JF815548 & Malaysia & This study \\
\hline FeLV-UPM26 & JF815549 & Malaysia & This study \\
\hline FeLV-UPM27 & JF815550 & Malaysia & This study \\
\hline FeLV-UPM28 & JF815551 & Malaysia & This study \\
\hline FeLV-UPM29 & JF815552 & Malaysia & This study \\
\hline FeLV-914MG & EU048352 & Brazil & GenBank \\
\hline FeLV-1235MG & EU43664 & Brazil & GenBank \\
\hline FeLV-843MG & DQ821500 & Brazil & GenBank \\
\hline FeLV-887MG & DQ821501 & Brazil & GenBank \\
\hline FeLV-922MG & EU48356 & Brazil & GenBank \\
\hline FeLV-1286MG & EU090948 & Brazil & GenBank \\
\hline FeLV-328MG & AY745878 & Brazil & GenBank \\
\hline FeLV-Rickard & AF052723 & USA & GenBank \\
\hline FeLV-FAIDS & M18247 & USA & GenBank \\
\hline FeLV-GM1 & D13922 & UK & GenBank \\
\hline FeLV-K01803 & K01803 & UK & GenBank \\
\hline FeLV-TWK25 & GQ465833 & Taiwan & GenBank \\
\hline FeLV-TW-K30 & GQ327961 & Taiwan & GenBank \\
\hline
\end{tabular}

Note: FeLV01-FeLV14 sequences were amplified from plasma viral RNA while the remaining local sequence (FeLVUPM13-FeLVUPM29) were amplified from proviral DNA.

detectable viraemia $[28,29]$. Our findings are consistent with the results of an earlier study that failed to isolate FeLV from about $10 \%$ of p 27 antigen-positive cats, irrespective of the antigen detection methods used. Such cats were considered as 
TABLE 2: Predicted transcription binding motifs found within the LTR-gag sequence of Malaysian FeLV isolates.

\begin{tabular}{|c|c|c|c|c|c|}
\hline Motifs & Sequence & Seq length & Coverage & Occurrence in local sequence & Occurrence in reference FeLV \\
\hline NF-GMa & GAGGTTTCAT & 10 & $523-532$ & All local seq except UPM08 & FeLV-Rickard; FeLV-FAIDs \\
\hline E1A-F & CGGATGT & 7 & $521-527$ & UPM08 & NA \\
\hline E1A-F & CGGATGT & 7 & $70-76$ & UPM18 & NA \\
\hline ELP & CAAGGTC & 7 & $523-527$ & UPM03, 14, 18, 20 & NA \\
\hline Sp1 & GGGGCTAGG & 7 & $521-527$ & UPM03, 18, 20 & NA \\
\hline$C \backslash$ EBPbeta & CTGGAAA & 7 & $387-393$ & UPM18, 20 & NA \\
\hline $\begin{array}{l}\text { Krox-20/WT1I- } \\
\text { del2 }\end{array}$ & CGCCCCCGC & 9 & $374-382$ & All local seq & FeLV-Rickard; FeLV-FAIDs \\
\hline $\mathrm{E} 2 \mathrm{~F}$ & TTTTGGCGG & 9 & $334-342$ & UPM03, 14, 18, 20 & FeLV-Rickard; FeLV-FAIDs \\
\hline BAF1 & TCCTTGTATACG & 12 & $301-312$ & All except UPM03, 14, 18, 20 & NA \\
\hline BAF1 & TCCTTGTATACG & 12 & $158-169$ & All local seq & NA \\
\hline AP-2 & CCCAACCG & 8 & $243-250$ & All local seq & FeLV-Rickard; FeLV-FAIDs \\
\hline AP-2 & CCCAACCG & 8 & $59-66$ & All local seq except UPM17 & FeLV-Rickard; FeLV-FAIDs \\
\hline AP-2 & CCCAACCG & 8 & $58-65$ & UPM03, 14, 18, 20, 25 & NA \\
\hline AP-2 & CCCAACCG & 8 & 4 to 11 & All except UPM03, 14 & NA \\
\hline AP-2 & CCCAACCG & 8 & 3 toll & All local seq & FeLV-Rickard \\
\hline GCF & CCGCCCC & 7 & $93-99$ & All local seq & FeLV-Rickard, FeLV-FAIDs \\
\hline GCF & CCGGCGC & 7 & $64-70$ & $\begin{array}{l}\text { All local seq except UPM03, } \\
\text { UPM } 14\end{array}$ & NA \\
\hline GCF & GCGCGCC & 7 & $26-32$ & UPM27 & NA \\
\hline HNF-3 & TGTTTGC & 7 & 129-135 & All local seq except UPM06 & NA \\
\hline PEA3 & GCGGAAGT & 8 & $69-76$ & UPM18 & NA \\
\hline TBP & TATAAAA & 7 & $39-45$ & All local seq & FeLV-Rickard, FeLV-FAIDs \\
\hline TFIIF-beta & TATAAAA & 7 & $39-45$ & All local seq & FeLV-Rickard, FeLV-FAIDs \\
\hline TRF & TATAAAA & 7 & $39-45$ & All local seq & FeLV-Rickard, FeLV-FAIDs \\
\hline TFIID & TATAAAA & 7 & $39-45$ & All local seq & FeLV-Rickard, FeLV-FAIDs \\
\hline TFIID & CTTCTCGC & 8 & 10 to 17 & UPM03, 14, 20 & NA \\
\hline MEP-1 & TATAAAA & 7 & $23-29$ & $\begin{array}{l}\text { All local seq except UPM03, } 14 \text {, } \\
18,20\end{array}$ & FeLV-Rickard \\
\hline MBF-I & TATAAAA & 7 & $23-29$ & $\begin{array}{l}\text { All local seq except UPM03, } 14 \text {, } \\
18,21\end{array}$ & FeLV-Rickard \\
\hline MTF-1 & TATAAAA & 7 & $23-29$ & All except UPM03, 14, 18, 22 & FeLV-Rickard \\
\hline
\end{tabular}

NA: not applicable or not present; seq: sequences.

"discordant," suggesting that p27 antigen-positive status may not always correlate with viraemia [30]. Another potential explanation for p27-positive-RNA-negative status might be false positive antigen or false negative RNA tests that arise occasionally because of low positive predictive value of p27 antigen tests in regions with low FeLV prevalence [31]. Clinical relevance of atypical FeLV infection is not well-understood, and it has been recommended to monitor the status of discordant cats over time $[27,28]$. No additional follow-up was carried out in the present study, because most owners were not willing to subject cats to repeated venepunctures [4].

Based on the U3LTR and partial gag regions, nucleotide sequence analyses revealed homology of $91-100 \%$ among Malaysian FeLV isolates. However, homology decreased to $84.6 \%$ when local isolates were compared with reference isolates. Previous studies reported strong sequence conservation
(>97\%) among FeLV isolates of different geographic and temporal clusters [32, 33]. In agreement with Jackson et al. [17], we do observe point mutations and nucleotide deletion in Malaysian FeLV isolates (see Supplementary Material available online at http://dx.doi.org/10.1155/2014/760961).While U3LTR is conserved in FeLV, field isolates have been reported to exhibit sequence variation within the terminally repeated LTRs regions $[17,33]$. Mutational changes in the LTR regions have been implicated with enhanced transcriptional and/or insertion activities of FeLV, thus supporting T-cell lymphomagenesis [34, 35].

In this study, several transcription binding motifs were predicted within the amplified U3LTR-gag region (Table 2). Of these, NF-GMa, Krox-20/WT1I-del2, BAF1, AP-2, TBP, TFIIF-beta, TRF, and TFIID motifs were found to be conserved between local FeLV isolates and the two characterized FeLV-Rickard subgroup A and FeLV-FAIDS reference 


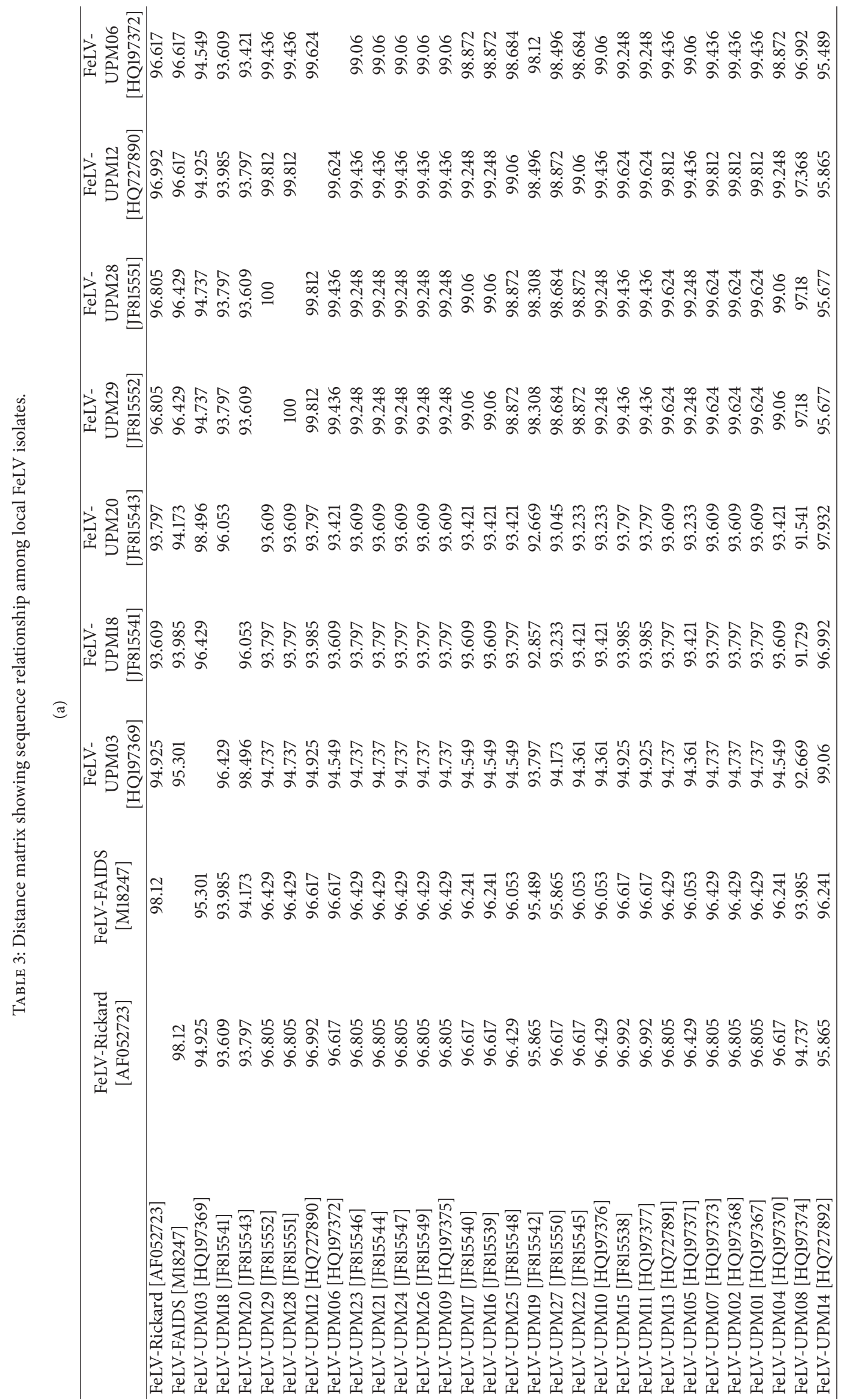




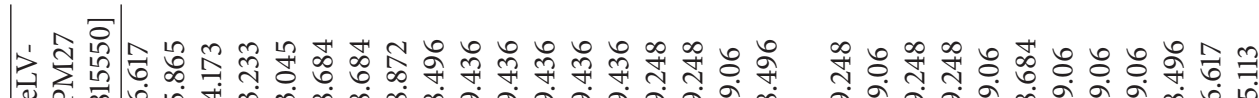

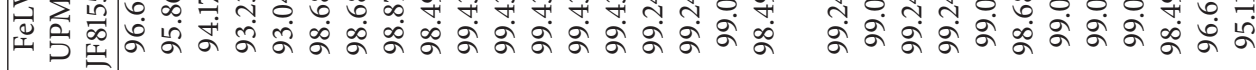

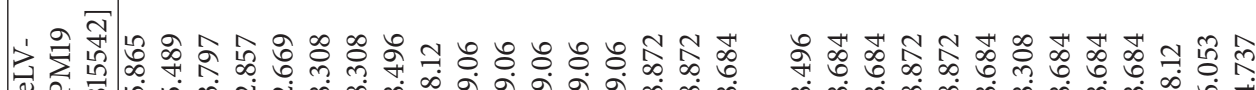

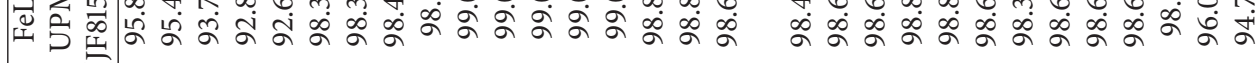

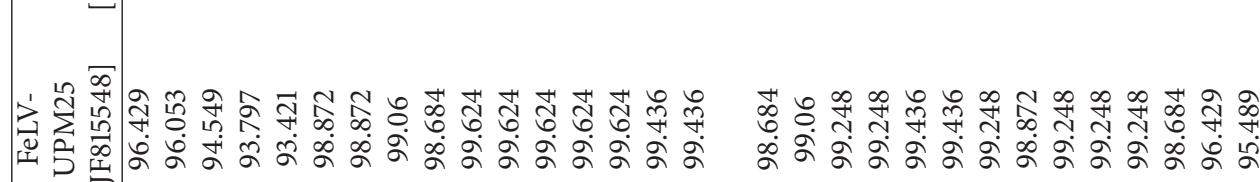

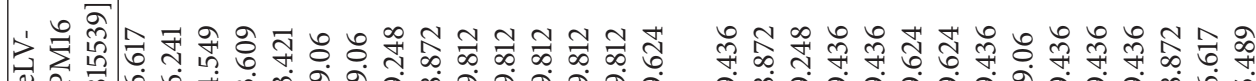
गे

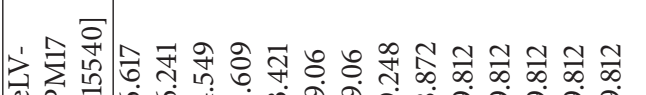

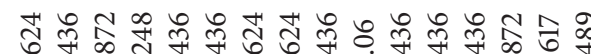

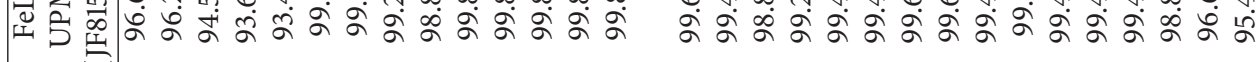

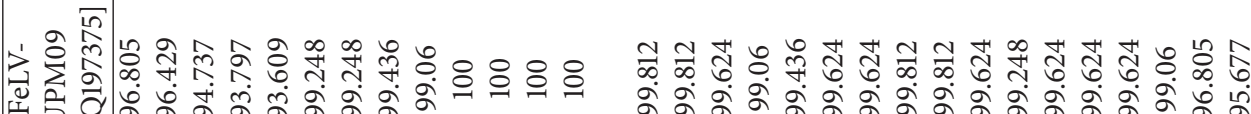

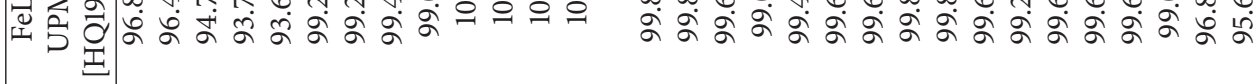

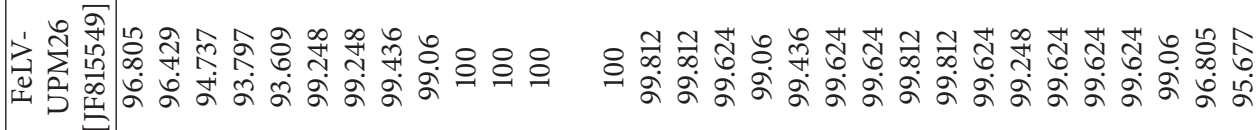

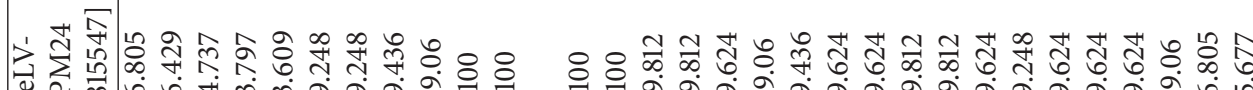
गे

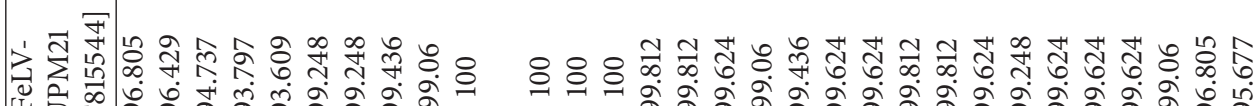
गे

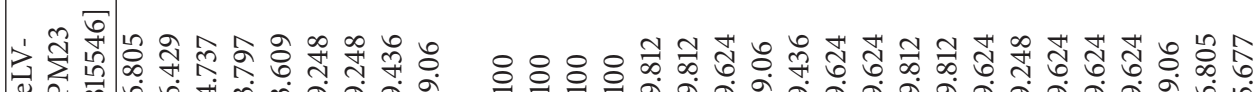
गे बूँ

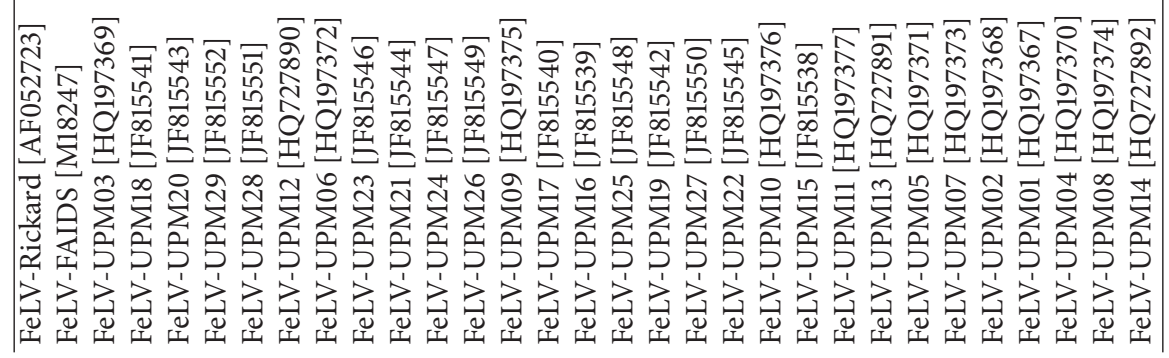




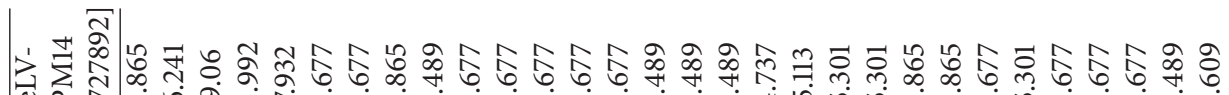
语言

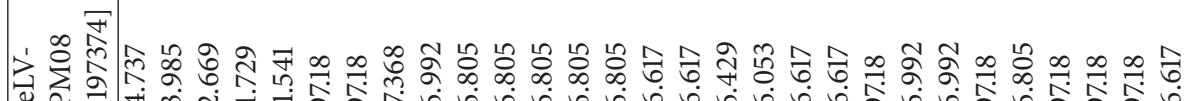

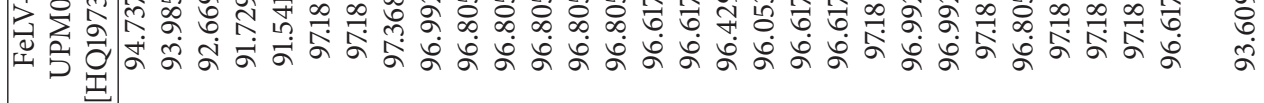

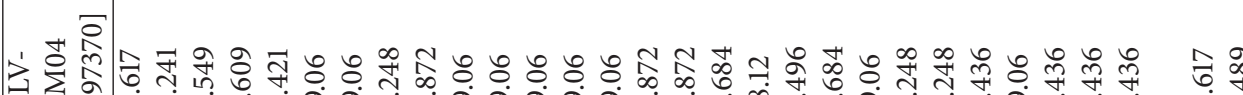

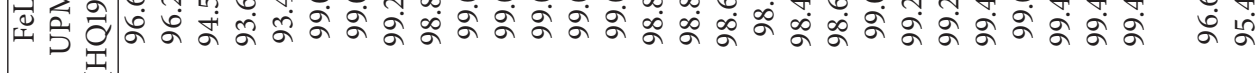

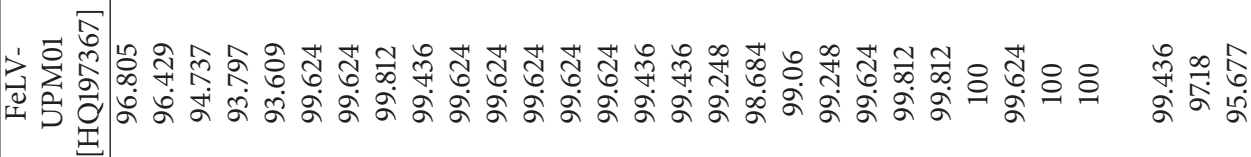

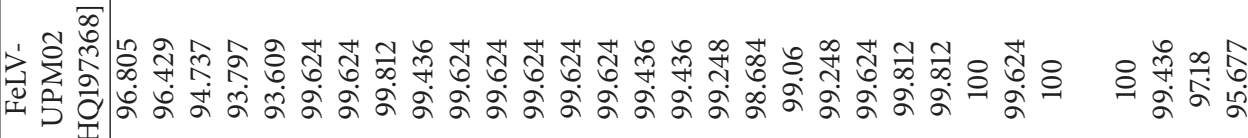

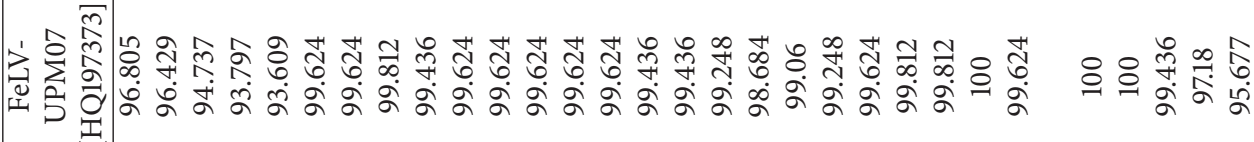

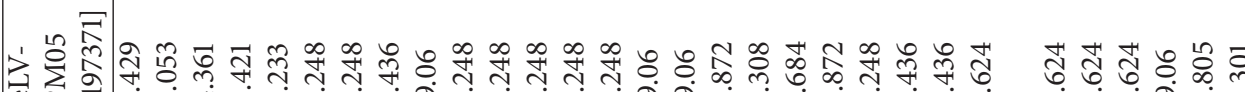

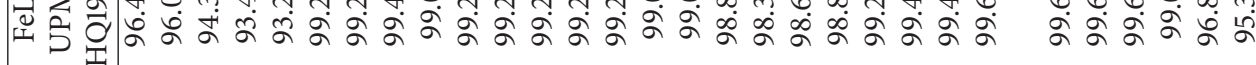

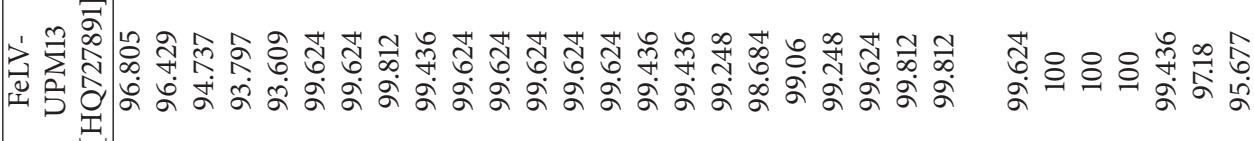

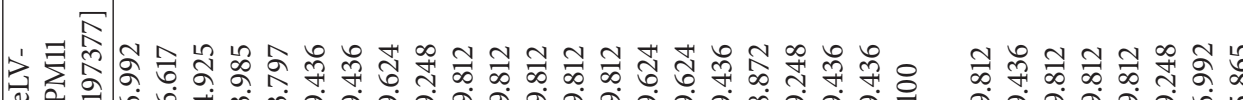

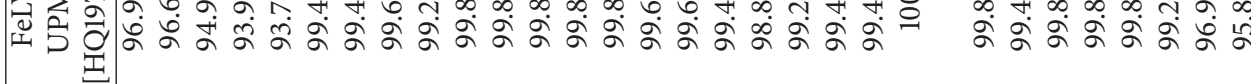

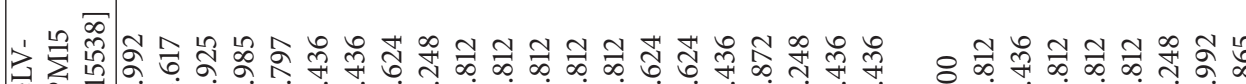
एँ

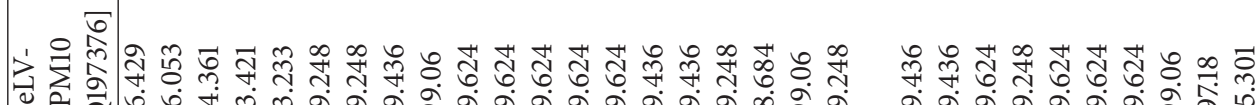
ए

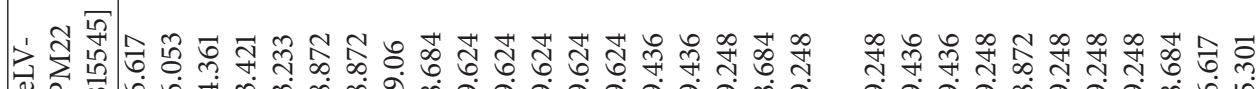
एँ

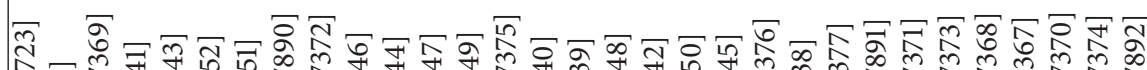

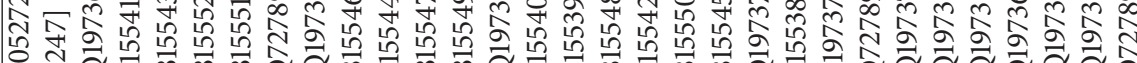

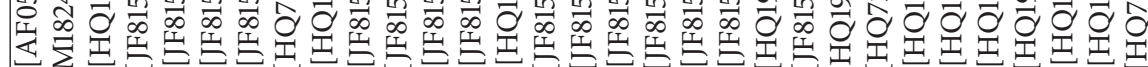
作

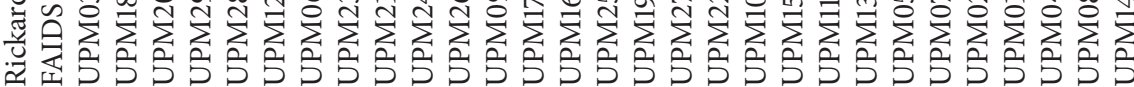

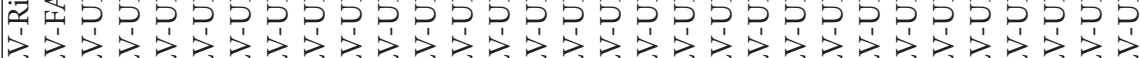

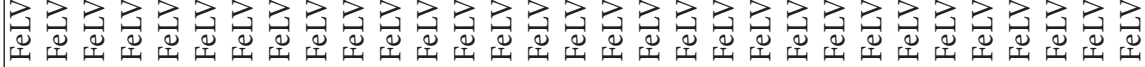




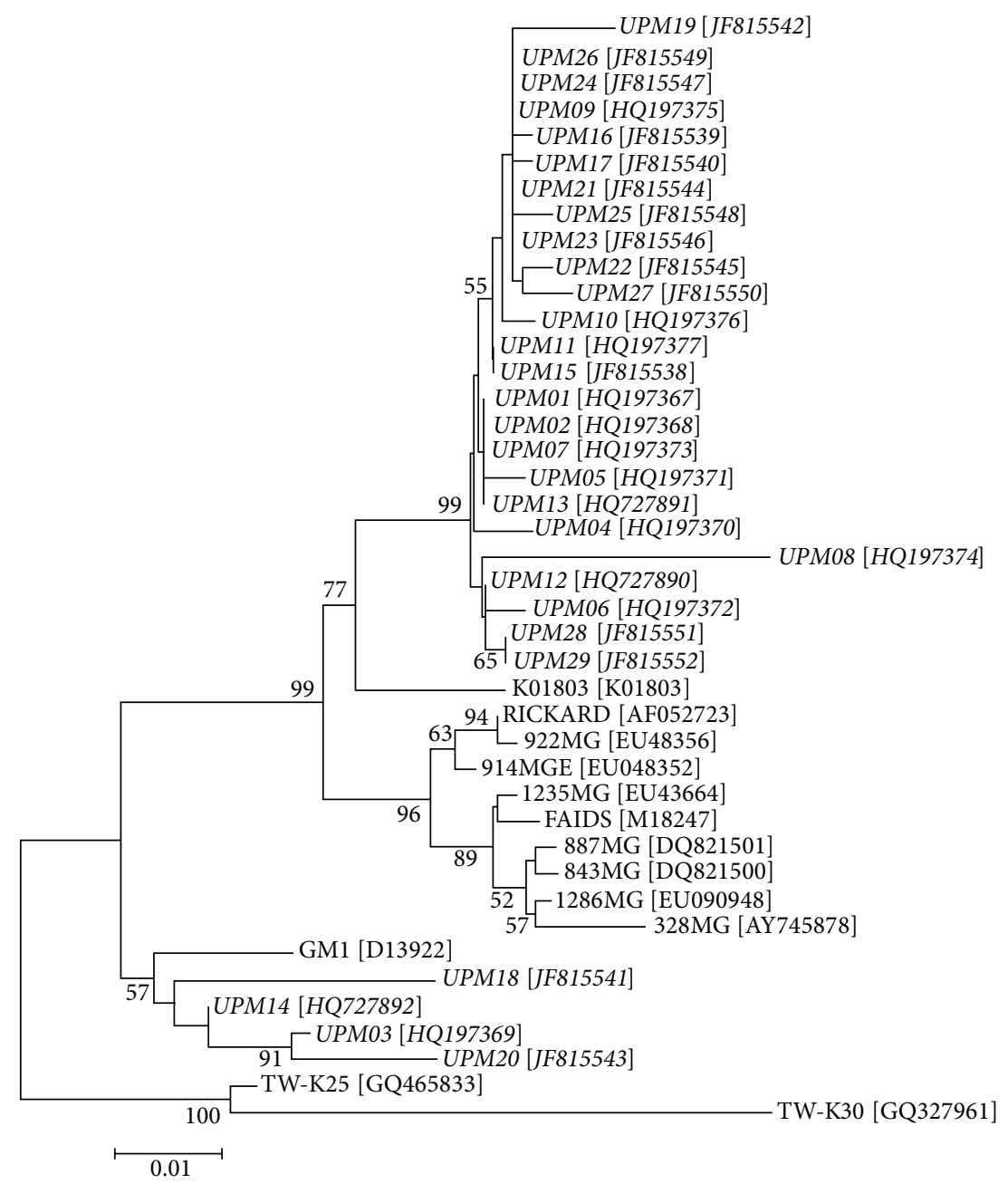

Figure 2: Neighbour-joining phylogenetic relationship of Malaysian FeLV isolates. The percentage of replicate trees in which the associated taxa clustered together in the bootstrap test (100 replicates) is shown next to the branches. Malaysian FeLV isolates are indicated in italic. Evolutionary analyses were conducted in MEGA5.

isolates. On the other hand, E1A-F, ELP, Sp1, C\EBPbeta, BAF1, GCF, HNF-3, and PEA3 motifs are found in some local isolates but were absent in reference sequences. These motifs may have implication for viral oncogenicity or probably favours viral replication. For example, an Spl enhancer, a member of Sp/Kruppel-like factor, was reported to activate gene transcription and contribute to abnormal metabolism of cancer cells $[36,37]$ whereas $C \backslash E B P b e t a$ regulates the growth and differentiation of myeloid as well as lymphoid cells [38]. Studies have shown that, the U3-LTR sequence contains multiple transcription binding sites that aid viral replication and pathogenesis. Interactions of different transcription binding factors, via the U3-LTRs, may contribute to cellular gene transactivation and viral leukemogenesis $[39,40]$. Enhancer motifs observed in this study appeared in multiple locations such as in the case of E1A-F, BAF1, and TFIID, each occurring in duplicate; GCF appeared in triplicate while AP-2 is repeated 5 times at different positions. An enhancer duplication and triplication has been reported in naturally occurring cases of FeLV-induced T-cell lymphomas
$[41,42]$. The clinical relevance of multiple enhancers in cats used in the present study is not determined, although some FeLV positive cats had evidence of different tumour forms at post-mortem (result not shown). Previous studies reported that E1A-F, a member ets-oncogene family transcription factor, upregulates the multiple matrix metalloproteinase (MMP) genes thus contributing to the malignant phenotypic activity by increasing the invasion and metastatic activities of cancerous cells [43]. TFIID, a potential protooncogene with TATA-box protein and a TBP-associated factor also plays role in transcription initiation and genome expression [44]. On the other hand, AP2 and SP1 are known to activate epidermal growth factor receptor (EGFR) gene. In addition, overexpression of these gene has been reported to cause cellular transformation $[45,46]$. Surprisingly we also identified a triplicate of GCF binding factor that has suppressor effect on EGR gene; these discrepancies, however, need further elucidation with quantitative real-time PCR [47].

Absence of length mutation (nucleotide position 473481) in Malaysian FeLV isolates, as observed in FeLV isolates 
from Taiwan (FeLV-TW-25 and FeLV-TW-30) and a European isolate (FeLV-GM1), might suggest limited influence of geography in evolutionary patterns of FeLV, unlike its lentiviral counterpart, feline immunodeficiency virus [33, 48].

Phylogenetic analysis based on the U3LTR-gag sequence revealed that Malaysian FeLV isolates are closely related (Tables 3(a), 3(b), and 3(c)) but when compared with reference isolates, separated into two distinct clusters, with the majority (86.2\%) being closely related to FeLV-K01803 isolate from UK. The remaining local FeLV isolates (13.8\%) clustered with FeLV-GM1 (Figure 2). The reason for the observed similarity between local FeLV isolates and European isolates, but not with Taiwanese isolates, may suggest the lack of geographical influence, this should be explored further. It is possible also that FeLV might have been introduced into Malaysia as a result of translocation of domestic pets from Europe. Due to a somewhat conserved nature of the U3LTR region, conclusion about the FeLV subgroup requires further investigations of FeLV envelope protein gene.

\section{Conclusion}

This study revealed the occurrence of FeLV viral RNA and provirus DNA among naturally infected Malaysian cats. Based on the U3LTR-gag sequence, Malaysian FeLV isolates are highly conserved and more closely related to K01803 isolate from UK compared to Taiwanese and other reference isolates. Presence of multiple enhancers some of which have been linked with FeLV induced tumours may contribute to the development of poor prognostic outcome in naturally infected Malaysian cats although this needs further investigation. Overall, this is the first molecular study for evidence of FeLV in Malaysia. We also identified several motifs that have potential implications in FeLV-induced leukemogenesis. Future studies need to explore association between FeLV positive status and occurrence of feline tumour in Malaysian cats. The present findings is useful in designing molecular diagnostics for clinical applications and for improved understanding of FeLV infection outcome and epidemiology.

\section{Conflict of Interests}

The authors declare that there is no conflict of interests regarding the publication of this paper.

\section{Acknowledgments}

The authors thank cat owners and clinicians for their support, Dr. Dennis F. Lawler for proof reading the paper, and Saeid Kadkhodaei for assistance in sequence analysis. This project was funded by Science Fund, Ministry of Science and Technology and Innovation, Project no. 02-01-04-SF1070.

\section{References}

[1] J. L. Rojko, E. A. Hoover, L. E. Mathes, R. G. Olsen, and J. P. Schaller, "Pathogenesis of experimental feline leukemia virus infection," Journal of the National Cancer Institute, vol. 63, no. 3, pp. 759-768, 1979.

[2] O. Jarrett, "The relevance of feline retroviruses to the development of vaccines against HIV," AIDS Research and Human Retroviruses, vol. 12, no. 5, pp. 385-387, 1996.

[3] S. E. Gleich, S. Krieger, and K. Hartmann, "Prevalence of feline immunodeficiency virus and feline leukaemia virus among client-owned cats and risk factors for infection in Germany," Journal of Feline Medicine and Surgery, vol. 11, no. 12, pp. 985992, 2009.

[4] F. Bande, S. S. Arshad, L. Hassan et al., "Prevalence and risk factors of feline leukaemia virus and feline immunodeficiency virus in peninsular Malaysia," BMC Veterinary Research, vol. 8, article 33, 2012.

[5] T. Miyazawa, Y. Ikeda, K. Maeda et al., "Seroepidemiological survey of feline retrovirus infections in domestic and leopard cats in Northern Vietnam in 1997," Journal of Veterinary Medical Science, vol. 60, no. 11, pp. 1273-1275, 1998.

[6] M. Chew-Lim, N. Fong, and S. Y. Chong, "A survey of the feline leukaemia virus in Singapore," Annals of the Academy of Medicine Singapore, vol. 18, no. 6, pp. 646-648, 1989.

[7] S. Maruyama, H. Kabeya, R. Nakao et al., "Seroprevalence of Bartonella henselae, toxoplasma gondii, FIV and FeLV infections in domestic cats in Japan," Microbiology and Immunology, vol. 47, no. 2, pp. 147-153, 2003.

[8] A. Litster and P. Nilkumhang, "Prevalence of feline leukaemia virus and feline immunodeficiency virus infection in Thailand," in Proceedings of the 28th Congress of World Small Animal Association, Bangkok, Thailand, 2003.

[9] J. A. Lin, M. C. Cheng, Y. Inoshima et al., "Seroepidemiological survey of feline retrovirus infections in cats in Taiwan in 1993 and 1994," The Journal of Veterinary Medical Science, vol. 57, no. 1, pp. 161-163, 1995.

[10] K. L. Gibson, K. Keizer, and C. Golding, "A trap, neuter, and release program for feral cats on Prince Edward Island," Canadian Veterinary Journal, vol. 43, no. 9, pp. 695-698, 2002.

[11] I. T. Lee, J. K. Levy, S. P. Gorman, P. C. Crawford, and M. R. Slater, "Prevalence of feline leukemia virus infection and serum antibodies against feline immunodeficiency virus in unowned free-roaming cats," Journal of the American Veterinary Medical Association, vol. 220, no. 5, pp. 620-622, 2002.

[12] S. Little, W. Sears, J. Lachtara, and D. Bienzle, "Seroprevalence of feline leukemia virus and feline immunodeficiency virus infection among cats in Canada," Canadian Veterinary Journal, vol. 50, no. 6, pp. 644-648, 2009.

[13] Y. M. Al-Kappany, M. R. Lappin, O. C. H. Kwok, S. A. AbuElwafa, M. Hilali, and J. P. Dubey, "Seroprevalence of Toxoplasma gondii and concurrent Bartonella spp., Feline immunodeficiency virus, feline leukemia virus, and Dirofilaria immitis infections in egyptian cats," Journal of Parasitology, vol. 97, no. 2, pp. 256-258, 2011.

[14] K. Hartmann, P. Griessmayr, B. Schulz et al., "Quality of different in-clinic test systems for feline immunodeficiency virus and feline leukaemia virus infection," Journal of Feline Medicine and Surgery, vol. 9, no. 6, pp. 439-445, 2007.

[15] T. Miyazawa and O. Jarrett, "Feline leukaemia virus proviral DNA detected by polymerase chain reaction in antigenaemic but non-viraemic ("discordant") cats," Archives of Virology, vol. 142, no. 2, pp. 323-332, 1997.

[16] V. Cattori and R. Hofmann-Lehmann, "Absolute quantitation of feline leukemia virus proviral DNA and viral RNA loads by 
TaqMan real-time PCR and RT-PCR," in Anonymous Molecular Beacons: Signalling Nucleic Acid Probes, Methods, and Protocols, p. 73, 2008.

[17] M. L. Jackson, D. M. Haines, and V. Misra, "Sequence analysis of the putative viral enhancer in tissues from 33 cats with various feline leukemia virus-related diseases," Veterinary Microbiology, vol. 53, no. 3-4, pp. 213-225, 1996.

[18] A. Amer, A. Siti Suri, O. Abdul Rahman et al., "Isolation and molecular characterization of type I and type II feline coronavirus in Malaysia," Virology Journal, vol. 9, article 278, 2012.

[19] T. A. Hall, "BioEdit: a user-friendly biological sequence alignment editor and analysis program for windows 95/98/NT," Nucleic Acids Symposium, vol. 41, pp. 95-98, 1999.

[20] M. Kearse, R. Moir, A. Wilson et al., "Geneious Basic: an integrated and extendable desktop software platform for the organization and analysis of sequence data," Bioinformatics, vol. 28, no. 12, pp. 1647-1649, 2012.

[21] J. Felsenstein, "Confidence limits on phylogenies: an approach using the bootstrap," Evolution, vol. 39, no. 4, pp. 783-791, 1985.

[22] F. M. Coelho, M. R. Q. Bomfim, F. D. A. Caxito et al., "Naturally occurring feline leukemia virus subgroup A and B infections in urban domestic cats," Journal of General Virology, vol. 89, no. 11, pp. 2799-2805, 2008.

[23] R. Hofmann-Lehmann, J. B. Huder, F. Boretti, B. Sigrist, and H. Lutz, "Feline leukaemia provirus load during the course of experimental infection and in naturally infected cats," Journal of General Virology, vol. 82, no. 7, pp. 1589-1596, 2001.

[24] B. R. Madewell and O. Jarrett, "Recovery of feline leukaemia virus from non-viraemic cats," Veterinary Record, vol. 112, no. 15, pp. 339-342, 1983.

[25] L. J. Gabor, M. L. Jackson, B. Trask, R. Malik, and P. J. Canfield, "Feline leukaemia virus status of Australian cats with lymphosarcoma," Australian Veterinary Journal, vol. 79, no. 7, pp. 476-481, 2001.

[26] S. Nesina, A. K. Helfer-Hungerbuehler, F. Boretti et al., "Transmission of FeLV infection by provirus positive blood," in Proceedings of the 11th International Feline Retrovirus Research Symposium Anonymous, Leipzig, Germany, August 2012.

[27] H. Lutz, D. Addie, S. Belák et al., "Feline leukaemia ABCD guidelines on prevention and management," Journal of Feline Medicine and Surgery, vol. 11, no. 7, pp. 565-574, 2009.

[28] E. A. Hoover and J. I. Mullins, "Feline leukemia virus infection and diseases," Journal of the American Veterinary Medical Association, vol. 199, no. 10, pp. 1287-1297, 1991.

[29] K. A. Hayes, J. L. Rojko, and L. E. Mathes, "Incidence of localized feline leukemia virus infection in cats," The American Journal of Veterinary Research, vol. 53, no. 4, pp. 604-607, 1992.

[30] O. Jarrett, M. C. Golder, and K. Weijer, "A comparison of three methods of feline leukaemia virus diagnosis," Veterinary Record, vol. 110, no. 14, pp. 325-328, 1982.

[31] J. A. Beatty, S. Tasker, O. Jarrett et al., "Markers of feline leukaemia virus infection or exposure in cats from a region of low seroprevalence," Journal of Feline Medicine and Surgery, vol. 13, no. 12, pp. 927-933, 2011.

[32] P. R. Donahue, E. A. Hoover, G. A. Beltz et al., "Strong sequence conservation among horizontally transmissible, minimally pathogenic feline leukemia viruses," Journal of Virology, vol. 62 , no. 3, pp. 722-731, 1988.

[33] C. Chandhasin, P. Lobelle-Rich, and L. S. Levy, "Feline leukaemia virus LTR variation and disease association in a geographical and temporal cluster," Journal of General Virology, vol. 85, no. 10, pp. 2937-2942, 2004.

[34] Y. Fujino, K. Ohno, and H. Tsujimoto, "Molecular pathogenesis of feline leukemia virus-induced malignancies: insertional mutagenesis," Veterinary Immunology and Immunopathology, vol. 123, no. 1-2, pp. 138-143, 2008.

[35] L. S. Levy, "Advances in understanding molecular determinants in FeLV pathology," Veterinary Immunology and Immunopathology, vol. 123, no. 1-2, pp. 14-22, 2008.

[36] M. C. Archer, "Role of sp transcription factors in the regulation of cancer cell metabolism," Genes and Cancer, vol. 2, no. 7, pp. 712-719, 2011.

[37] R. Pal, M. Janz, D. L. Galson et al., " $\mathrm{C} / \mathrm{EBP} \beta$ regulates transcription factors critical for proliferation and survival of multiple myeloma cells," Blood, vol. 114, no. 18, pp. 3890-3898, 2009.

[38] A. Amer, A. Siti Suri, O. Abdul Rahman et al., "Isolation and molecular characterization of type I and type II feline coronavirus in Malaysia," Virology Journal, vol. 9, article 278, 2012.

[39] S. K. Ghosh and D. V. Faller, "Feline leukemia virus long terminal repeat activates collagenase IV gene expression through AP-1," Journal of Virology, vol. 73, no. 6, pp. 4931-4940, 1999.

[40] A. L. Abujamra, D. V. Faller, and S. K. Ghosh, "Mutations that abrogate transactivational activity of the feline leukemia virus long terminal repeat do not affect virus replication," Virology, vol. 309, no. 2, pp. 294-305, 2003.

[41] Y. Matsumoto, Y. Momoi, T. Watari, R. Goitsuka, H. Tsuilmoto, and A. Hasegawa, "Detection of enhancer repeats in the long terminal repeats of feline leukemia viruses from cats with spontaneous neoplastic and nonneoplastic diseases," Virology, vol. 189, no. 2, pp. 745-749, 1992.

[42] G. B. Athas, P. Lobelle-Rich, and L. S. Levy, "Function of a unique sequence motif in the long terminal repeat of feline leukemia virus isolated from an unusual set of naturally occurring tumors," Journal of Virology, vol. 69, no. 6, pp. 3324-3332, 1995.

[43] M. Shindoh, F. Higashino, and T. Kohgo, "E1AF, an etsoncogene family transcription factor," Cancer Letters, vol. 216, no. 1, pp. 1-8, 2004.

[44] M. Purrello, C. Di Pietro, A. Viola et al., "Genomics and transcription analysis of human TFIID," Oncogene, vol. 16, no. 12, pp. 1633-1638, 1998.

[45] R. Kageyama, G. T. Merlino, and I. Pastan, "Nuclear factor ETF specifically stimulates transcription from promoters without a TATA box," Journal of Biological Chemistry, vol. 264, no. 26, pp. 15508-15514, 1989.

[46] T. J. Velu, L. Beguinot, W. C. Vass et al., "Epidermal growth factor-dependent transformation by a human EGF receptor proto-oncogene," Science, vol. 238, no. 4832, pp. 1408-1410, 1987.

[47] Y. Kitadai, H. Yamazaki, W. Yasui et al., "GC factor represses transcription of several growth factor/receptor genes and causes growth inhibition of human gastric carcinoma cell lines," Cell Growth \& Differentiation, vol. 4, no. 4, pp. 291-296, 1993.

[48] J. Pecon-Slattery, J. L. Troyer, W. E. Johnson, and S. J. O’Brien, "Evolution of feline immunodeficiency virus in Felidae: implications for human health and wildlife ecology," Veterinary Immunology and Immunopathology, vol. 123, no. 1-2, pp. 32-44, 2008. 

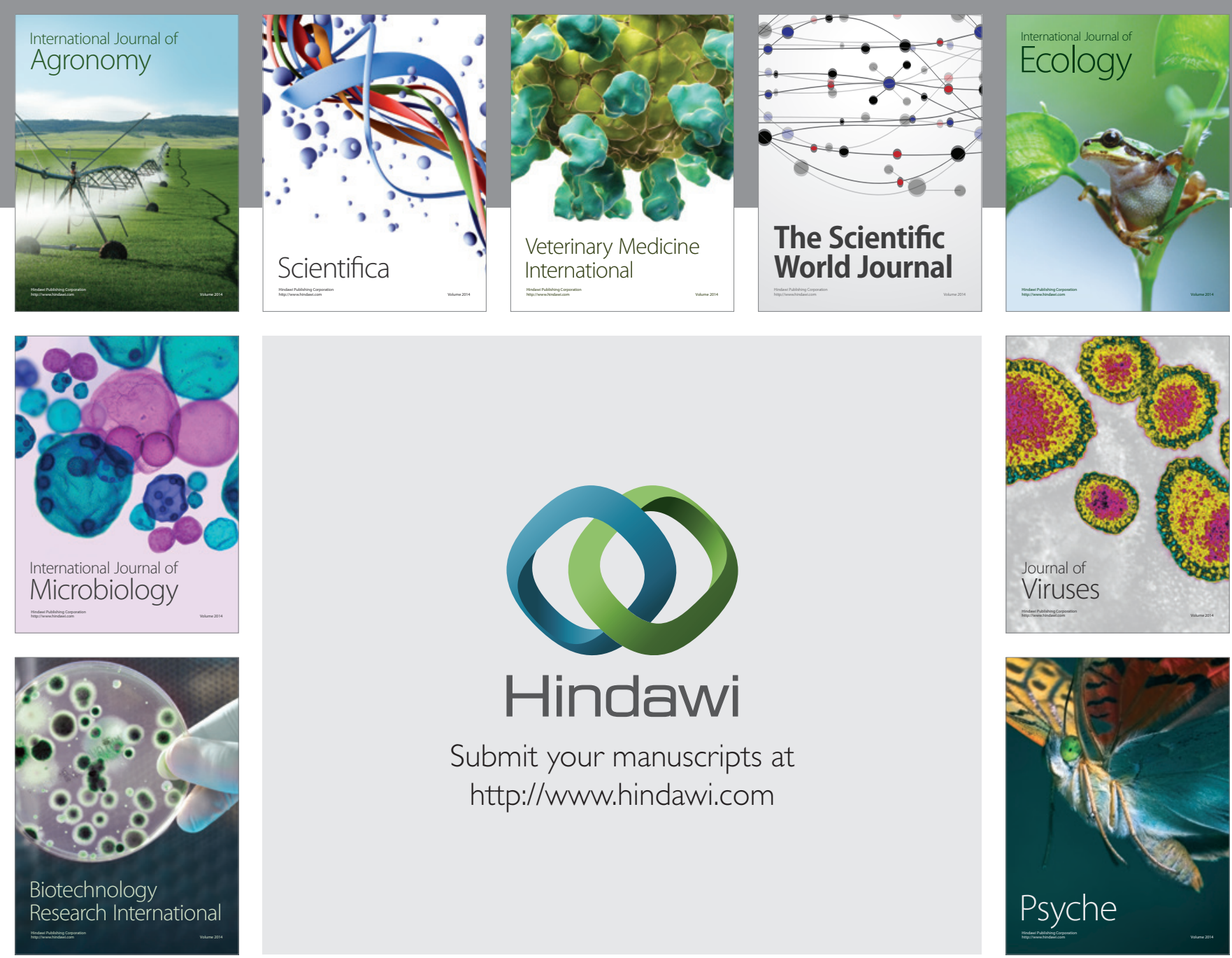

Submit your manuscripts at http://www.hindawi.com
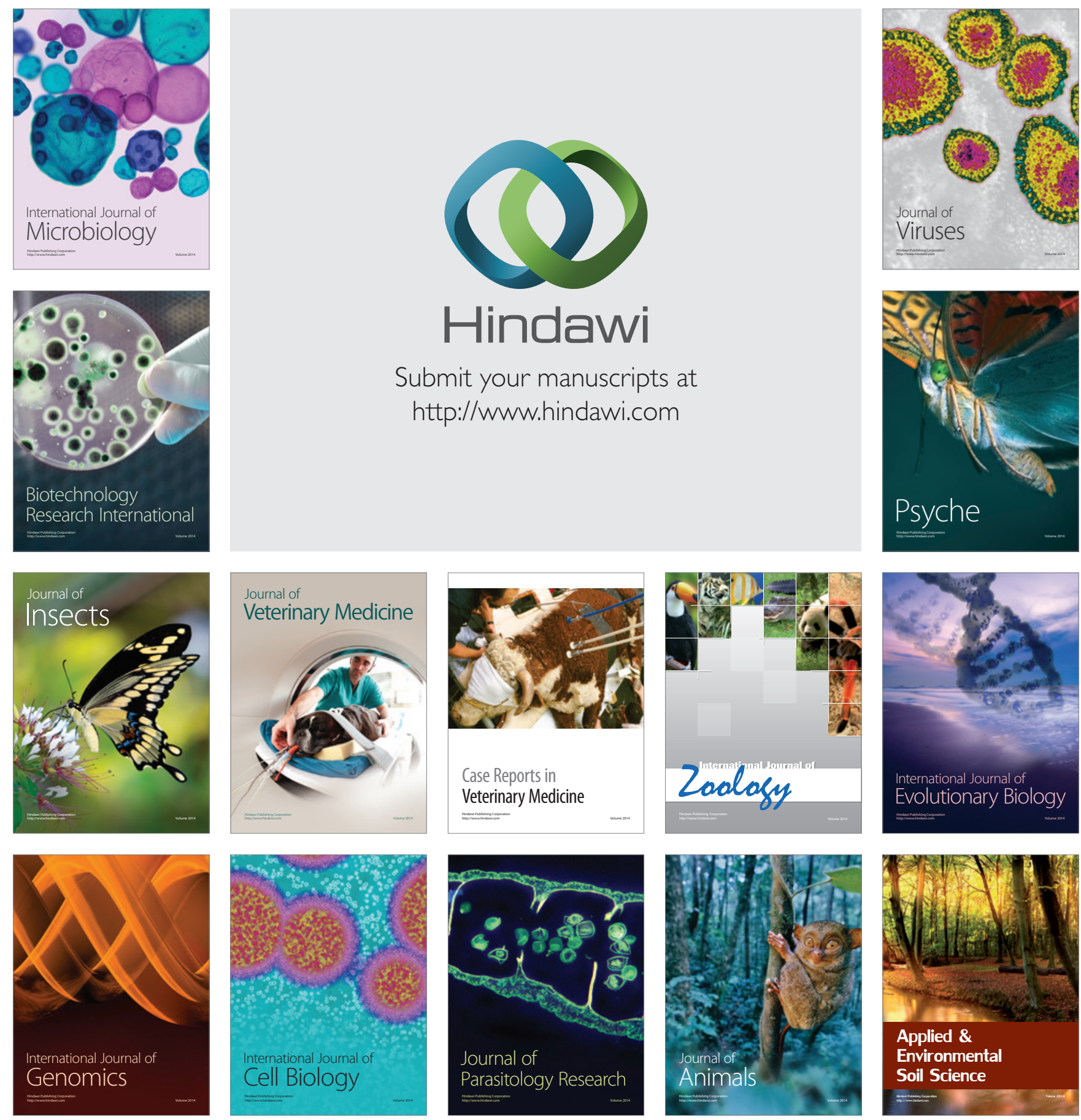